\title{
Synthesis and Characterization of Molybdenum Nitride Hydrodenitrogenation Catalysts
}

Jeong-Gil Choi, James R. Brenner, Craig W. Colling, Brian G. Demczyk, Judy L. Dunning and Levi T. Thompson*

Department of Chemical Engineering, University of Michigan, Ann Arbor, Michigan 48109-2136

\begin{abstract}
Details concerning the relationships between the structural, chemical and catalytic properties of Mo nitrides have been elucidated. A series of Mo nitride catalysts were prepared by the temperature programmed reaction of $\mathrm{MoO}_{3}$ with $\mathrm{NH}_{3}$. The structural properties of these nitrides were complex functions of the heating rates and space velocities employed. Two reaction sequences were proposed to account for the synthesis of high, medium and low surface area materials. An interesting conclusion was that the degree of reduction of the molybdate precursor or intermediate governed the structural properties of the product. Some evidence is also presented to suggest that the nucleation and growth rates involved in the transformation of the oxide to the nitride were significantly influenced by the synthesis conditions. The Mo nitrides proved to be exceptional pyridine hydrodenitrogenation catalysts. Their catalytic properties were superior to those of a commercial sulfided Co-Mo hydrotreatment catalyst, having higher activities and better $C-N$ bond hydrogenolysis selectivities. Hydrodenitrogenation over the Mo nitrides appeared to be structure-sensitive. While detailed relationships between the catalytic activity and surface stoichiometry could not be ascertained, there did appear to be a correlation between the activity, and the particle size and grain boundary length. We proposed that at least two types of HDN sites existed on the Mo nitride surfaces; modest activity sites on the particles and high activity sites at grain boundaries. The N/Mo stoichiometry of the highest activity catalyst was near unity suggesting that $\mathrm{MoN}$ was present perhaps localized at the grain boundaries. Finally structures near or at the surface were markedly different from those of the bulk. While the predominant bulk phase was $\gamma-$ $\mathrm{Mo}_{2} \mathrm{~N}$, the surface appeared to consist of either non-stoichiometric $\beta-\mathrm{Mo}_{16} \mathrm{~N}_{7}$ or mixtures of $\mathrm{Mo}$ and $\mathrm{\beta}-\mathrm{Mo}_{16} \mathrm{~N}_{7}$.
\end{abstract}

\section{INTRODUCTION}

Advances in the preparation of high surface area early transition metal nitrides and carbides have sparked interest in their development as catalysts and as supports [1-3]. Molybdenum and tungsten nitrides and carbides in particular have received a great deal of attention due to their competitive activities for reactions including hydrodenitrogenation $[4,5], \mathrm{CO}$ hydrogenation [6], and $\mathrm{NH}_{3}$ synthesis [7]. In addition, their function as catalysts for hydrocarbon dehydrogenation, hydrogenolysis and isomerization 
reactions resembles that of platinum [8-10]. The catalytic and adsorptive properties of nitrides and carbides are governed by their bulk and surface structure and stoichiometry. While there is some information concerning the effects of bulk composition on the catalytic properties of these materials, there is currently very little known about the effects of surface structure and stoichiometry.

In this paper we discuss the results of our efforts to elucidate the structural and chemical character of the active species in Mo nitride hydrodenitrogenation (HDN) catalysts. These catalysts were prepared using the temperature programmed reaction (TPR) technique, an effective method for balancing the rates of transformations that occur during solid state reactions. Hydrodenitrogenation was selected as the test reaction because it is one of the most promising prospects for the commercial utilization of Mo nitride and carbide catalysts. Schlatter et al. [5] reported that high surface area Mo nitrides and carbides were as active as a commercial sulfided Ni$\mathrm{Mo} / \mathrm{Al}_{2} \mathrm{O}_{3}$ hydrotreatment catalyst. More importantly, the selectivity for $\mathrm{C}-\mathrm{N}$ bond hydrogenolysis in preference to aromatic ring saturation was significantly higher for the nitride and carbide catalysts. The sulfided molybdate catalysts that are currently used for hydrotreating crude apparently remove organic nitrogen only after the associated aromatic ring is saturated. In severe cases more than half of the $\mathrm{H}_{2}$ is consumed in saturating the aromatic ring. The expense of $\mathrm{H}_{2}$ provides a significant economic incentive for the further investigation of Mo nitride and carbide HDN catalysts. The development of new and improved hydrotreatment catalysts is also spurred by the relatively high nitrogen and sulfur content of domestic crude, and by Clean Air Act legislation requiring reduced pollutant emission levels.

\section{EXPERIMENTAL}

\subsection{Synthesis}

The $2^{\mathrm{k}}$ factorial experimental design strategy was used to guide our synthesis of the Mo nitride catalysts. This scheme allowed us to qualitatively and quantitatively assess the influence of the heating rates and space velocity on the properties of the catalysts. A series of eight catalysts were synthesized via the TPR of $\mathrm{MoO}_{3}\left(99.95 \%\right.$, Alfa) with $\mathrm{NH}_{3}(99.998 \%$, Scott). Initially the sample was linearly heated from room temperature to $623 \mathrm{~K}$ over approximately 30 minutes. The temperature was increased from 623 to $723 \mathrm{~K}$ at 40 or $100 \mathrm{~K} / \mathrm{hr}$ during the first linear heating segment $\left(\beta_{1}\right)$. The temperature was then increased from 723 to $973 \mathrm{~K}$ at 100 or $200 \mathrm{~K} / \mathrm{hr}$ during the second linear heating segment $\left(\beta_{2}\right)$, and held at $973 \mathrm{~K}$ for an additional hour. The intermediate temperature was selected based on results reported by Jaggers et al. [11] indicating that $\mathrm{MoO}_{3}$ reacts with $\mathrm{NH}_{3}$ to produce either a Mo oxynitride or $\mathrm{MoO}_{2}$ at $720 \mathrm{~K}$ on its route to the Mo nitride final product. The molar hourly space velocity (SV), defined as the ratio of the molar flow rate of $\mathrm{NH}_{3}$ to the moles of $\mathrm{MoO}_{3}$, was 8.5 or $17 \mathrm{hr}^{-1}$. These conditions are similar to those used previously to produce high surface area Mo nitrides [12]. After synthesis, the furnace was opened and the product was rapidly cooled in the flowing $\mathrm{NH}_{3}$. Once the system cooled to room temperature the product was purged with $\mathrm{He}$ for 10 minutes then passivated in a mixture of $0.996 \% \mathrm{O}_{2}$ in $\mathrm{He}$ (Scott) flowing at $20 \mathrm{~cm}^{3} / \mathrm{min}$. This passivation was continued for 2 hours. After passivation, the product was taken out of the reactor, and stored in a desiccator for subsequent evaluation. 


\subsection{Sorption Analyais}

The surface areas were measured by the single point BET method using a Quantasorb Model QS-17 sorption analyzer. The standard pretreatment consisted of heating the sample in $\mathrm{H}_{2}$ (99.99\%, Air Products) at $673 \mathrm{~K}$ for 3 hours in order to remove the passivation layer and other impurities. The temperatures and times were varied for selected samples to evaluate the effects of the pretreatment conditions on the surface area. The maximum temperature available using our Quantasorb was $673 \mathrm{~K}$. A mixture of $29.3 \%$ $\mathrm{N}_{2}$ in $\mathrm{He}$ (Scott) was used as the adsorbate gas. Accurately measured pulses ( \pm $2 \%$ ) of purified $\mathrm{N}_{2}(99.998 \%$, Air Products) were used to calibrate the instrument. The average size of particles at the surface, $d_{p}$, was estimated using the following equation

$d_{p}=f_{g} / S_{g} \rho$

where $f_{\mathrm{s}}$ is a constant characteristic of the particle shape (here taken to be 6 for spherical and cubic particles), $S_{g}$ is the BET surface area, and $p$ is the density of the material (here taken to be $9.4 \mathrm{gr} / \mathrm{cm}^{3}$ for $\gamma$-Mo2N). The use of this equation presumes that the nitrides were non-porous (electron microscopic results confirm non-porous character).

Oxygen chemisorption measurements were made by the pulsed method also using the Quantasorb sorption analyzer. Oxygen chemisorption has been demonstrated to be a useful method for determining the number of sites in unpromoted Mo sulfide hydrotreatment catalysts [13, 14]. By analogy we expected $\mathrm{O}_{2}$ chemisorption would be useful for characterizing Mo nitride HDN catalysts. The pretreatment conditions were basically the same as those used in advance of determining the BET surface areas. After pretreatment, the catalysts were purged with flowing $\mathrm{He}$ at room temperature. Calibrated volumes of $9.98 \% \mathrm{O}_{2}$ in $\mathrm{He}$ (Scott) were then injected into the $\mathrm{He}$ carrier gas entering the catalyst bed. The $\mathrm{O}_{2}$ that was not adsorbed was detected and recorded. When the $\mathrm{O}_{2}$ peaks reached essentially constant area, the surface was assumed to be saturated. The amount of oxygen adsorbed was easily calculated from the number of pulses and the peak areas. Replicate experiments were performed for both BET surface area and $\mathrm{O}_{2}$ uptake, and the reported values are averages of these replicates.

\subsection{X-ray Diffraction}

Crystalline phase identification and texturing analysis were accomplished using a computer controlled Rigaku DMAX-B x-ray diffractometer. The average crystallite sizes, $d_{c}$, were calculated using the Scherrer equation [15]:

$d_{c}=K \lambda(B \cos \theta)$

where $K$ is a constant (here taken to be 0.9 ), $\lambda$ is the wavelength of the $x$ radiation (1.5405 $A$ ), $B$ is the corrected peak width, and $\theta$ is the Bragg angle of the diffraction peak. The peak width was taken to be the full-width at halfmaximum intensity of the most intense peak in the pattern. Lattice constants were calculated using the interplanar spacings obtained from the $x$-ray diffraction (XRD) patterns.

\subsection{Activity Measurements}

Pyridine HDN activity measurements were carried out using a $6.35 \mathrm{~mm}$ OD Pyrex glass flow reactor. Approximately $200 \mathrm{mg}$ of catalyst was loaded into the reactor over a plug of glass wool and heated in $\mathrm{H}_{2}$ from room temperature to 
$673 \mathrm{~K}$ at a rate of $2 \mathrm{~K} / \mathrm{min}$. The catalysts were held at $673 \mathrm{~K}$ for at least 12 hours then cooled to the reaction temperature. Between activity measurements, the catalysts were regenerated in $\mathrm{H}_{2}$ at $673 \mathrm{~K}$ for at least 12 hours. A commercial Co-Mo/ $\mathrm{Al}_{2} \mathrm{O}_{3}$ hydrotreatment catalyst (Katalco 477) consisting of 8-11\% $\mathrm{Mo}$ and $1-4 \%$ Co supported on $\gamma-\mathrm{Al}_{2} \mathrm{O}_{3}$ was also evaluated. This catalyst was initially reduced in $\mathrm{H}_{2}$ under conditions similar to those used for the Mo nitrides then sulfided at $673 \mathrm{~K}$ in a $2 \% \mathrm{H}_{2} \mathrm{~S}_{\mathrm{H}_{2}}$ mixture for 4 hours followed by rereduction in $\mathrm{H}_{2}$ at $673 \mathrm{~K}$ for 12 hours. The $\mathrm{H}_{2}(99.99 \%$, Air Products) was purified during passage through a commercial oxygen trap (Matheson) and a bed of activated carbon and molecular sieves. Routine experiments were carried out at temperatures between 588 and $648 \mathrm{~K}$ and atmospheric pressure using 20 $\mathrm{cm}^{3} / \mathrm{min}$ of flowing $\mathrm{H}_{2}$ saturated with pyridine $(99+\%$, Aldrich) at $273 \mathrm{~K}$ (P $\mathrm{Pyp}=$ 4.54 torr). The products were separated using a Hayesep D packed column (Alltech Associates) and analyzed using a HP 5890 gas chromatograph equipped with both flame ionization and thermal conductivity detectors. The retention times and peak areas were calibrated using standard alkane and alkene mixtures (Scott Specialty Gases, $\pm 2 \%$ ).

\subsection{Temperature Programmed Desorption of $\mathbf{H}_{2}$}

An Altamira AMI-M characterization system equipped with a thermal conductivity detector was used to perform $\mathrm{H}_{2}$ temperature programmed desorption (TPD). The system was controlled and data was acquired using an IBM PC/XT compatible computer. Between 100 to $250 \mathrm{mg}$ of the catalyst was placed in a Pyrex U-tube over a glass wool plug. The thermocouple was placed into the bed to insure good thermal contact with the catalyst.

Pretreatment of the catalysts consisted of a two hour reduction in flowing $\mathrm{H}_{2}$ (99.999\%, Scott) at $673 \mathrm{~K}$ followed by a one hour flush in Ar to remove any residual adsorbed hydrogen. The heating rate preceding these pretreatments was arbitrarily set at $20 \mathrm{~K} / \mathrm{min}$. The catalyst was then cooled to room temperature in flowing Ar (99.998\%, Scott) followed by exposure to $\mathrm{H}_{2}$ for 30 minutes at room temperature. A final Ar flush for 10 minutes was expected to remove any weakly adsorbed hydrogen. During desorption, the temperature was increased linearly from room temperature to $673 \mathrm{~K}$.

Five TPD spectra were collected for each catalyst. Three experiments were conducted at a heating rate of $15 \mathrm{~K} / \mathrm{min}$ using Ar flow rates of 10,20 , and 40 $\mathrm{cc} / \mathrm{min}$. Spectra were also collected at heating rates of 25 and $35 \mathrm{~K} / \mathrm{min}$ using a flow rate of $10 \mathrm{cc} / \mathrm{min}$. By independently varying the flow rate and the heating rate, the number and types of sites could be determined.

\subsection{High Resolution Transmission Flectron Microscopy}

Samples for high resolution transmission electron microscopy (HRTEM) were prepared by gently grinding and ultrasonically dispersing the Mo nitrides in isopropanol. A drop of this suspension was placed onto a holey carbon coated $3 \mathrm{~mm} \mathrm{Cu}$ mesh grid. The microscopy was performed at $400 \mathrm{kV}$, using a JEOL 4000 EX transmission electron microscope, with a point-to-point resolution of $\leq 2 \AA$. Subsequent analysis included digitizing the negatives into version $1.35 \mathrm{NIH}$ images using a Cohu Series 4810 CCD camera, modified to incorporate a Hartley Fast Fourier Transform [16] routine for diffractogram analysis from very small regions $(\leq 5 \mathrm{~nm})$.

\subsection{X-ray Photoelectron Spectroscopy}

Samples for $x$-ray photoelectron spectroscopy (XPS) analysis were prepared by pressing $\sim 200 \mathrm{mg}$ of catalyst into a self-supporting wafer. The spectroscopy was carried out using a Phi ESCA 5400 spectrometer and the $\mathrm{Mg}_{\mathrm{g}} \mathrm{K}$-ray 
source. Spectra of the catalysts were collected before and after reduction in flowing $\mathrm{H}_{2}$ (99.99\%, Air Products) at $673 \mathrm{~K}$ for 3 hours using an in situ reactor. Vacuum in the test chamber during transfer of the catalyst from the in situ reactor and during the collection of spectra was typically less than $3 \times 10^{-\theta}$ torr. The spectrometer energies were calibrated using the $A u 4 f_{1 / 2}$ peak at $84.0 \mathrm{eV}$ and the $\mathrm{Cu} 2 \mathrm{p}_{3 / 2}$ peak at $932.6 \mathrm{eV}$. Charging effects were accounted for by referencing the binding energies to that of adventitious carbon (284.8 eV). Gaussian and/or Lorentzian peaks were deconvoluted using a non-linear least-squares algorithm.

\section{RasuLTS}

\subsection{Sorption Analysis}

The BET surface areas of the Mo nitrides ranged up to $-100 \mathrm{~m}^{2} / \mathrm{gr}$. For convenience we categorized these materials as low $\left(<20 \mathrm{~m}^{2} / \mathrm{gr}\right)$, medium (20-80 $\mathrm{m}^{2} / \mathrm{gr}$ ) or high ( $>80 \mathrm{~m}^{2} / \mathrm{gr}$ ) surface area. Table 1 gives the BET surface areas for the Mo nitrides following pretreatment in $\mathrm{H}_{2}$ at $673 \mathrm{~K}$ for 3 hours and following exposure to the HDN reaction conditions. The surface area of the $\mathrm{MoO}_{3}$ precursor was $\sim 1 \mathrm{~m}^{2} / \mathrm{gr}$. Clearly the synthesis conditions influenced the surface areas. All other variables held constant, the lower the first heating rate, the higher the surface area. Consider for example the two nitrides prepared using a space velocity of $17 \mathrm{hr}^{-1}$ with a second heating rate of 200 $\mathrm{K} / \mathrm{hr}$. The material produced using the low first heating rate, MoN-2, had a surface area almost 5 times that of the material produced using the high heating rate, MoN-1. The only exception to this trend was for MoN-7 and MoN8. The influence of the second heating rate appeared to depend on the value of the first heating rate. For nitrides prepared using a first heating rate of 40 $\mathrm{K} / \mathrm{hr}$, increasing the second heating rate caused an increase in the surface area. When the reactants were first heated at $100 \mathrm{~K} / \mathrm{hr}$, the high second heating rate led to the lower surface area nitride.

Table 1

Effect of the Preparative Conditions on Surface Area

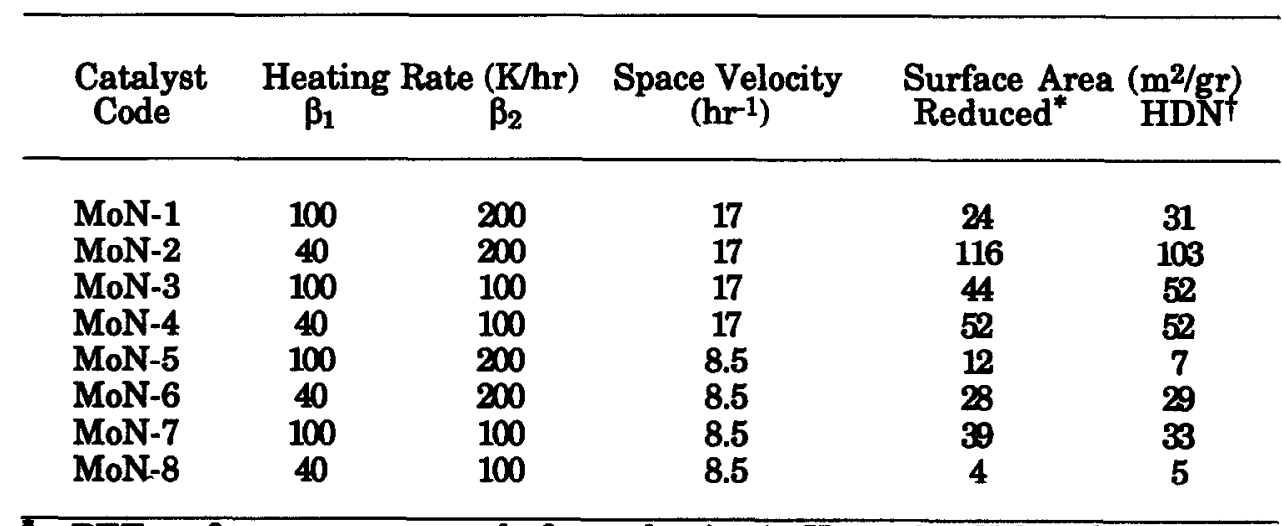

BET surface area measured after reduction in $\mathrm{H}_{2}$ at $673 \mathrm{~K}$ for 3 hours.

$\dagger$ BET surface area measured after HDN reaction rate measurements. 
Without exception, higher space velocities resulted in the production of materials with higher surface areas. This is consistent with previous studies which also indicated that high space velocities enhanced the ability to produce high surface area Mo nitrides and carbides [1, 2]. This behavior has been attributed to the adverse effects of $\mathrm{H}_{2} \mathrm{O}$ vapor. Water vapor, even in small concentrations, can cause hydrothermal sintering and the loss of surface area. High space velocities would facilitate the rapid removal of $\mathrm{H}_{2} \mathrm{O}$ from the vicinity of the surface, thus reducing the degree of hydrothermal sintering. It is also possible that $\mathrm{H}_{2} \mathrm{O}$ inhibits reactions that lead to the formation of higher surface area materials. Again high space velocities would be expected to minimize the concentration of $\mathrm{H}_{2} \mathrm{O}$ and other volatiles near the surface.

There was a nearly linear relationship between the $\mathrm{O}_{2}$ uptake and the BET surface area (Figure 1) indicating that oxygen was a nonselective adsorbate and the uptake merely reflected the physical character of the Mo nitrides. This conclusion is based partially on the observation that the surface characters of the Mo nitrides were different. Similar interpretations have been made for $\mathrm{O}_{2}$ chemisorption on $\mathrm{MoS}_{2}$ catalysts $[13,14]$.

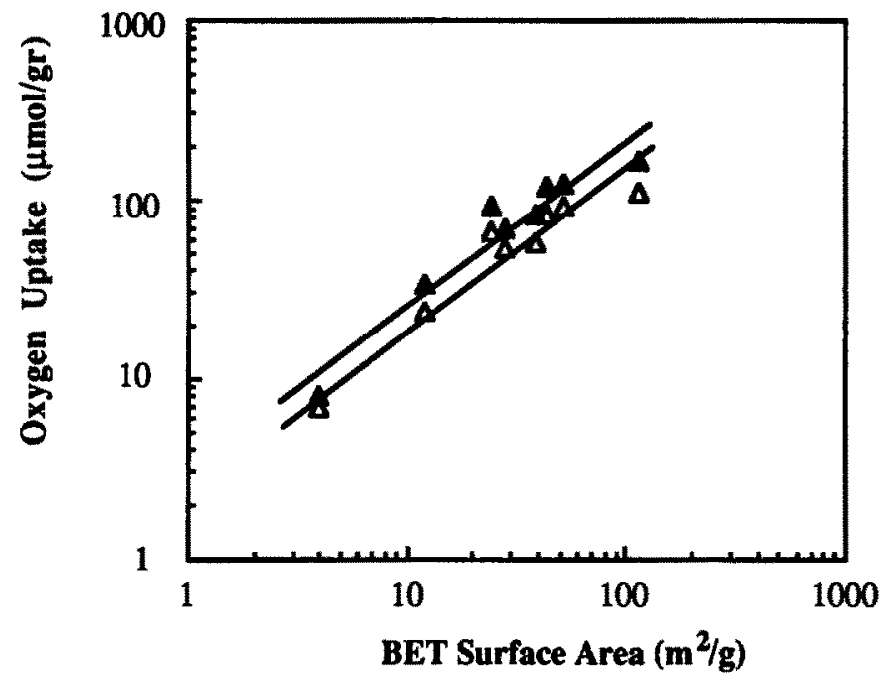

Figure 1. A comparison of the BET surface areas and $\mathrm{O}_{2}$ uptakes following pretreatment in flowing $\mathrm{H}_{2}$ at $673 \mathrm{~K}$ for three hours. The uptake at $298 \mathrm{~K}(\Delta)$ was $-30 \%$ greater than the uptake at $195 \mathrm{~K}(\Delta)$.

The $\mathrm{O}_{2}$ uptake at $298 \mathrm{~K}$ was greater than the uptake at $195 \mathrm{~K}$. The difference between these values could be due to either oxidation of subsurface layers or incomplete surface coverage. There is substantial evidence that oxygen can diffuse into the subsurface layers of Mo nitrides and carbides [17, 18]. During the passivation of Mo nitrides and carbides it is generally accepted that a thin passivating layer is formed during exposure to dilute $\mathrm{O}_{2}$ mixtures. Markel and Van Zee [17] indicated that the passivation layer under conditions similar to those used in our synthesis experiments was slightly thicker than one monolayer but did not produce a distinct oxide phase. The rates of oxygen 
diffusion into the subsurface and reaction with Mo would be accelerated by higher temperatures thus the $\mathrm{O}_{2}$ uptake should increase with increasing temperature if subsurface sites were involved. Furthermore, if subsurface sites were involved, the relative difference between the uptakes at 195 and 298 $\mathrm{K}$ should be constant and reproducible. In each case the $\mathrm{O}_{2}$ uptake at $298 \mathrm{~K}$ was approximately $30 \%$ larger than that at $195 \mathrm{~K}$. As a check we performed an $\mathrm{O}_{2}$ uptake measurement at $298 \mathrm{~K}$ immediately following the measurement at $195 \mathrm{~K}$. The additional $\mathrm{O}_{2}$ uptake amounted to the difference between the individual uptakes at 298 and $195 \mathrm{~K}$. Thus we concluded that oxygen diffused into the subsurface layer at least during chemisorption experiments carried out at $298 \mathrm{~K}$. We also believe that $\mathrm{O}_{2}$ dissociatively adsorbed on the surface of the Mo nitrides since molecular oxygen would be much too large to diffuse through the lattice.

Oxygen number densities were determined from the $\mathrm{O}_{2}$ uptakes and the BET surface areas (Table 2). We assumed that the $\mathrm{O}_{2}$ chemisorbed at $195 \mathrm{~K}$ was restricted to the surface. The $\mathrm{O}_{2}$ number density was nearly constant and averaged $0.11 \times 10^{15} \mathrm{O}_{2} / \mathrm{cm}^{2}$. Using the usual approximation that the surface consists of equal proportions of the main low-index planes, a Mo number density of $1.09 \times 10^{15} \mathrm{Mo} / \mathrm{cm}^{2}$ is expected for $\gamma$-Mo2N. Assuming a 1:1 $\mathrm{Mo}: 0$ stoichiometry, an uptake of $0.11 \times 10^{15} \mathrm{O}_{2} / \mathrm{cm}^{2}$ is equivalent to $\sim 21 \%$ surface coverage by atomic oxygen. Surface coverages assuming $0.146 \mathrm{~nm}^{2} / \mathrm{O}_{2}$ molecule were similar and averaged $\sim 16 \%$. The equivalent of $\sim 5 \%$ of a monolayer of oxygen appeared to reside in the subsurface following chemisorption at $298 \mathrm{~K}$. Volpe and Boudart [7] reported similar surface coverages for CO (10-30\%). Haddix et al. $[19,20]$ reported surface coverages of $\sim 10 \%$ for hydrogen and $\sim 40 \%$ for ammonia on a series of high surface area Mo nitrides. Hydrogen was believed to adsorb as localized rafts on nitrogen deficient patches of Mo on the surface.

Table 2

Summary of Chemisorption Results

Catalyst $\mathrm{O}_{2}$ Uptake ( $\mu \mathrm{mol} / \mathrm{gr}$ ) Code Q195 K $298 \mathrm{~K}$

$\mathrm{O}_{2}$ Number Density* Surface Coverage $\left(\times 10^{-15} / \mathrm{cm}^{2}\right.$ )

\begin{tabular}{lcccc}
\hline MoN-1 & 67 & 92 & 0.17 & 31 \\
MoN-2 & 112 & 165 & 0.06 & 11 \\
MoN-3 & 85 & 118 & 0.12 & 22 \\
MoN-4 & 93 & 123 & 0.11 & 20 \\
MoN-5 & 24 & 34 & 0.12 & 22 \\
MoN-7 & 54 & 70 & 0.13 & 24 \\
MoN-8 & 58 & 85 & 0.09 & 17 \\
\hline
\end{tabular}

* Based on $\mathrm{O}_{2}$ Uptake at $195 \mathrm{~K}$.

We evaluated the effects of $\mathrm{H}_{2}$ pretreatment temperature and time on the sorption properties. As the pretreatment temperature was increased up to 673 $\mathrm{K}$, the surface area and $\mathrm{O}_{2}$ uptake increased monotonically. We believe that 
this behavior was due to crevice opening caused by the progressive conversion of the oxynitride passivation layer into either the denser Mo nitride or Mo metal. The dissolution of oxygen into the Mo nitride during passivation could cause expansion of the lattice closing off some of the internal structure. Conversely, removal of oxygen from this oxynitride would cause contraction of the lattice and crevice opening. $\mathrm{A} \mathrm{H}_{2}$ pretreatment time of 3 hours at $673 \mathrm{~K}$ usually resulted in the highest surface area.

\subsection{Bulk Structure}

The bulk structure of the Mo nitride catalysts consisted primarily of $\gamma$ MogN. The low surface area catalysts also contained Mo while one of the medium surface area catalysts contained $\mathrm{MoO}_{2}$. Table 3 gives some of the structural features of the Mo nitrides.

For the high and medium surface area catalysts the crystallite and particle sizes were similar, however, the crystallites were much smaller than the particles in the low surface area catalysts, MoN-5 and MoN-8. Recall that the particle sizes were based on the surface area, therefore they reflect average surface character as compared to the crystallite sizes which reflect average bulk character. Deviations between the crystallite and particle sizes for these catalysts indicated either the presence of polycrystalline particles or differences between the surface and bulk structures. Similarities between the crystallite sizes estimated using XRD (bulk) and electron microscopy (surface) suggested that the particles were polycrystalline.

Table 3

Bulk Structural Properties of the Mo Nitrides

\begin{tabular}{|c|c|c|c|c|}
\hline $\begin{array}{l}\text { Catalyst } \\
\text { Code }\end{array}$ & $\begin{array}{c}\text { Particle Size } \\
(\AA)\end{array}$ & $\underset{(\AA)}{\text { Crystallite Size }}$ & $\begin{array}{l}\text { Phases } \\
\text { Present }\end{array}$ & $\mathrm{I}(200) / \mathrm{I}(111)$ \\
\hline $\begin{array}{l}\text { MoN-1 } \\
\text { MoN-2 } \\
\text { MoN-3 }\end{array}$ & $\begin{array}{c}266 \\
55 \\
145\end{array}$ & $\begin{array}{l}97 \\
77 \\
108 \\
448\end{array}$ & $\begin{array}{c}\gamma-\mathrm{Mo}_{2} \mathrm{~N} \\
\gamma-\mathrm{Mo}_{2} \mathrm{~N} \\
\gamma-\mathrm{Mo}_{2} \mathrm{~N} \\
\mathrm{MoO}_{2}\end{array}$ & $\begin{array}{l}0.6 \\
2.9 \\
0.8\end{array}$ \\
\hline $\begin{array}{l}\text { MoN-4 } \\
\text { MoN-5 }\end{array}$ & $\begin{array}{l}123 \\
532\end{array}$ & $\begin{array}{c}130 \\
78 \\
282\end{array}$ & $\begin{array}{c}\gamma-\mathrm{Mo}_{2} \mathrm{~N} \\
\gamma-\mathrm{Mo}_{2} \mathrm{~N} \\
\mathrm{Mo}_{0}\end{array}$ & $\begin{array}{l}1.1 \\
0.6\end{array}$ \\
\hline $\begin{array}{l}\text { MoN-6 } \\
\text { MoN-7 } \\
\text { MoN-8 }\end{array}$ & $\begin{array}{c}226 \\
140 \\
1596\end{array}$ & $\begin{array}{l}142 \\
132 \\
80 \\
266\end{array}$ & $\begin{array}{c}\gamma-\mathrm{Mo}_{2} \mathrm{~N} \\
\gamma-\mathrm{Mo}_{2} \mathrm{~N} \\
\gamma-\mathrm{Mo}_{2} \mathrm{~N} \\
\mathrm{Mo}\end{array}$ & $\begin{array}{l}0.8 \\
0.7 \\
0.7\end{array}$ \\
\hline$\gamma-\mathrm{Mo}_{2} \mathrm{~N}$ & & & & $0.48^{*}$ \\
\hline
\end{tabular}

From the JCPDS Files.

A significant degree of texturing was observed in the highest surface area catalysts as evidenced by the relative intensities in the diffraction patterns. The ratio of the intensities of the (200) and (111) reflections, $I(200) /(111)$, was used as a measure of the texturing of the $\gamma-\mathrm{Mo}_{2} \mathrm{~N}$ crystallites. For randomly distributed $\gamma$-Mo2 $\mathrm{N}$ crystallites of uniform dimensions $\mathrm{I}(200) / \mathrm{I}(111)$ should be 
0.5. The $I(200) / I(111)$ ratios for the two highest surface area catalysts, MoN-2 and MoN-4, were 2.9 and 1.1, respectively. This texturing remained following diminution by grinding and suggested the presence of $\gamma-\mathrm{Mo}_{2} \mathrm{~N}$ rafts extending out in the $<100\rangle$ directions. Texturing of this type appears characteristic of high surface area Mo nitrides $[11,21]$. The average lattice constants for the $\gamma$ $\mathrm{MO}_{2} \mathrm{~N}$ domains ranged from 4.16-4.20 A. The value given in the JCPDS tables for $\gamma-\mathrm{Mog}_{2} \mathrm{~N}$ is $4.163 \mathrm{~A}$. The lattice constant increased slightly with increasing surface area. In addition, the $\gamma-\mathrm{M}_{2} \mathrm{~N}$ crystallite sizes to some extent tracked with surface area. The $\gamma$-Mo2N crystallites in the medium surface area catalysts were greater than $100 \AA$ while the crystallites in the low and high surface area catalysts were $\sim \mathbf{8 0} \AA$.

\subsection{Catalytic Properties}

During the first 10 minutes on stream, the pyridine HDN reaction rates decreased after which the rates leveled off to a nearly constant value. The initial HDN rates were up to 2-fold higher than the steady-state rates measured after 2-3 hours on stream. The steady-state reaction rates could be maintained for an additional 12 hours with minimal decrease. The reaction rates during subsequent runs were reproducible to within $\pm 15 \%$. No change in the reaction rate was observed when the reactant flow rate was varied from 10 to $40 \mathrm{~cm}^{3} / \mathrm{min}$, which indicated the absence of external transport limitations. The steady-state reaction rates and activities of the Mo nitrides and the $\mathrm{Co}-\mathrm{Mo} / \mathrm{Al}_{2} \mathrm{O}_{3}$ catalyst are compared in Table 4. The nitrides were more active than the $\mathrm{Co}-\mathrm{Mo} / \mathrm{Al}_{2} \mathrm{O}_{3}$ catalyst on a per gram, per surface area, and per $\mathrm{O}_{2}$ uptake basis. Qualitatively similar results have been reported for other Mo nitride hydrodenitrogenation [5] and hydrodesulfurization [17] catalysts.

Table 4

Catalytic Reaction Rates and Parameters

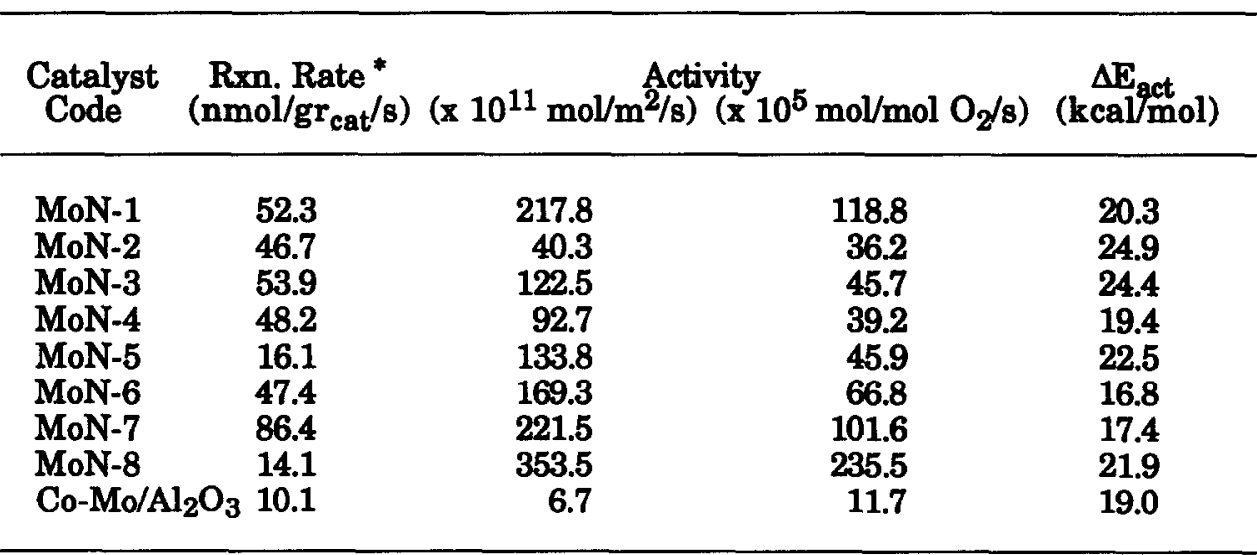

* Pyridine HDN reaction rates measured at $633 \mathrm{~K}$ and $\sim 101 \mathrm{kPa}$.

Normalizing the reaction rates exaggerated the difference between the activities of the nitrides. While there was no simple relationship between the HDN reaction rates and either the BET surface areas or $\mathrm{O}_{2}$ uptakes, the 
reaction rates on a per gram basis for the low surface area catalysts were similar and much lower than those of the other nitride catalysts. The activity appeared to be a function of particle size. With the exception of $\mathrm{MoN}-5$ and MoN-7, the activity increased with increasing particle size. Thus we concluded that HDN over the Mo nitrides was a structure-sensitive reaction. Markel and Van Zee [17] demonstrated that the low surface area (large particle size) Mo nitrides also had the highest specific hydrodesulfurization (HDS) activities. Structure-sensitivity is usually attributed to differences between the activities of the various crystallographic planes exposed at the catalyst surface. For catalysts like the Mo nitrides differences between the activities of differing phases and structures that can coexist on the catalyst surface must also be considered.

The product distributions for the Mo nitrides were similar and did not appear to be influenced by the structural properties of the catalysts. The products were almost exclusively alkanes and alkenes, with trace amounts of cyclopentane. Pentane was the most abundant product, followed by butane and pentene. Lower molecular weight hydrocarbons accounted for less than $5 \%$ of the product. The selectivities for the Mo nitride catalysts are compared to that of the $\mathrm{Co}-\mathrm{Mo} / \mathrm{Al}_{2} \mathrm{O}_{3}$ catalyst in Figure 2. The sulfided $\mathrm{Co}-\mathrm{Mo} / \mathrm{Al}_{2} \mathrm{O}_{3}$ catalyst produced mostly ethylene, ethane, propylene, and propane. No C5's were observed for this catalyst. The cracking activity may have been a consequence of the presence of acidic sites on the $\mathrm{Al}_{2} \mathrm{O}_{3}$. Nevertheless, the selectivities for $\mathrm{C}-\mathrm{N}$ bond hydrogenolysis over $\mathrm{C}-\mathrm{C}$ bond hydrogenolysis for the Mo nitrides were superior to that of the $\mathrm{Co}-\mathrm{Mo} / \mathrm{Al}_{2} \mathrm{O}_{3}$ catalyst.

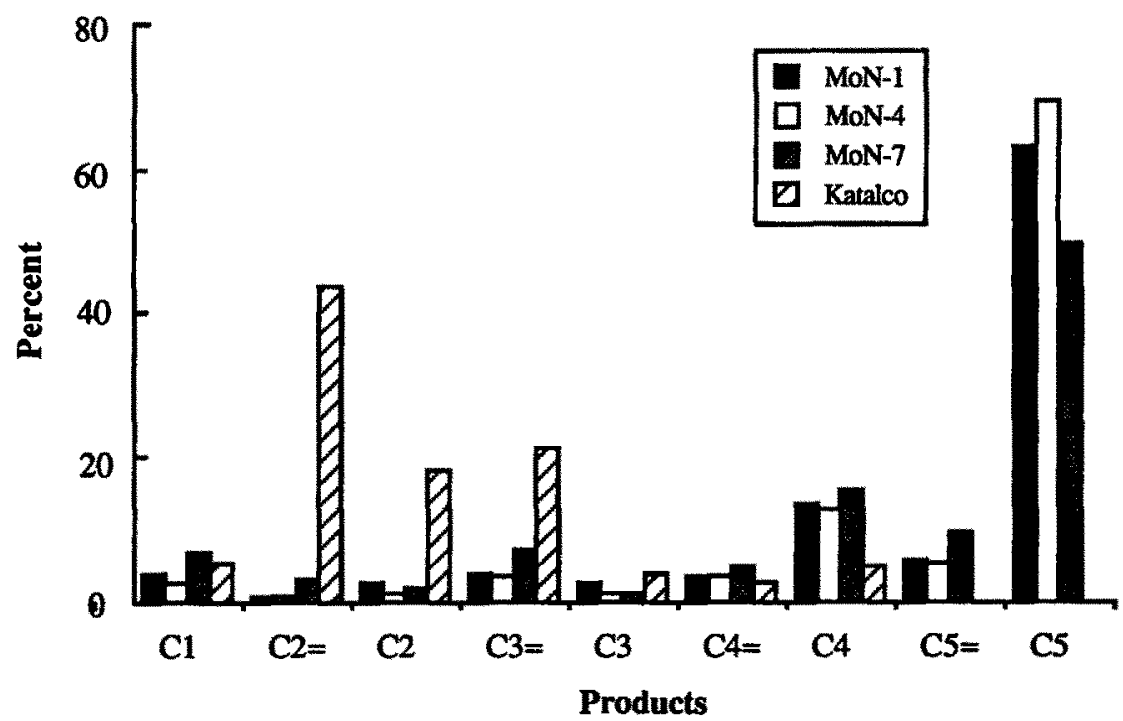

Figure 2. Product selectivities for the pyridine HDN over selected Mo nitride catalysts and the sulfided $\mathrm{Co}-\mathrm{Mo} / \mathrm{Al}_{2} \mathrm{O}_{3}$ (Katalco) catalyst. The $\mathrm{HDN}$ activities and selectivities were measured at $633 \mathrm{~K}$ and $-101 \mathrm{kPa}$. 
The product distributions also indicated a lower $\mathrm{H}_{2}$ economy for the Mo nitride catalysts than for the $\mathrm{Co}-\mathrm{Mo} / \mathrm{Al}_{2} \mathrm{O}_{3}$ catalyst. This contrasts a report by Schlatter et al. [5] that in the absence of organosulfur compounds the nitride catalysts had greater $\mathrm{H}_{2}$ economies than a commercial sulfided Ni-Mo hydrotreatment catalyst. They also found that when organosulfur in the form of $\mathrm{CS}_{2}$ was co-fed with quinoline, the product distribution approached that of the commercial hydrotreatment catalyst. In preliminary experiments we observed that the pyridine HDN selectivities for the nitrides in the presence of $\mathrm{H}_{2} \mathrm{~S}$ shifted toward the production of propylene and propane while the activity remained constant. This suggested that the nitride surfaces were partially sulfided during exposure to the organosulfur compounds. We are currently studying the influence of sulfur on the surface and catalytic properties of Mo nitrides.

\subsection{Temperature Programmed Desorption of $\mathrm{H}_{2}$}

Molybdenum nitrides are classified as interstitial compounds because the nitrogen resides at interstitial sites in the Mo lattice. Other small atoms like carbon and hydrogen can also occupy these interstitial sites. One might anticipate that hydrogen can migrate into the subsurface during exposure to $\mathrm{H}_{2}$ at elevated temperatures. Experimentally, desorption peaks produced by subsurface sites can be distinguished from those produced by surface sites by examining the effect of carrier gas flow rate and heating rate on the TPD spectrum. With increasing flow rate, peaks produced by multiple surface adsorption sites are expected to shift to lower temperatures, but their relative intensities should not change. Variations in the heating rate should also cause a shift in the peak temperatures without influencing the relative sizes of the peaks if the peaks are due to surface sites. A change in the relative peak intensities with varying flow rate or heating rate is believed to be evidence of subsurface sites [22].

Exemplary TPD spectra for the Mo nitrides are illustrated in Figures 3 and 4 , and the results are summarized in Table 5. All peak temperatures referred to in the table were taken from spectra collected using a heating rate of 15 $\mathrm{K} / \mathrm{min}$ and a carrier gas flow rate of $10 \mathrm{cc} / \mathrm{min}$. The error associated with determining the temperature for the maximum desorption rate was estimated to be $\pm 10 \mathrm{~K}$. For convenience we grouped the peaks into the following categories; low energy surface sites $\left(\pi_{L}\right)$, high energy surface sites $\left(\pi_{H}\right)$ or subsurface sites $\left(\pi_{\mathrm{S}}\right)$. Peaks with maximum intensities at temperatures less than $500 \mathrm{~K}$ were attributed to the low energy surface sites, while peaks with maximum intensities at temperatures greater than $500 \mathrm{~K}$ were attributed to the high energy sites. We assigned peaks whose relative intensities varied with flow rate and/or heating rate to subsurface sites. The associated surface desorption peak is always located at a lower temperature.

Many of the catalysts possessed both high and low energy surface sites. The MoN-2, MoN-3 and MoN-6 catalysts produced similar TPD spectra. These nitrides were among the least active catalysts. The catalyst with the highest specific activity, MoN-8, produced a unique TPD spectrum. This spectrum consisted of a single, very intense low temperature peak. These observations implied significant differences between the adsorptive properties which may correspond to differences between the catalytic properties of the nitrides. 


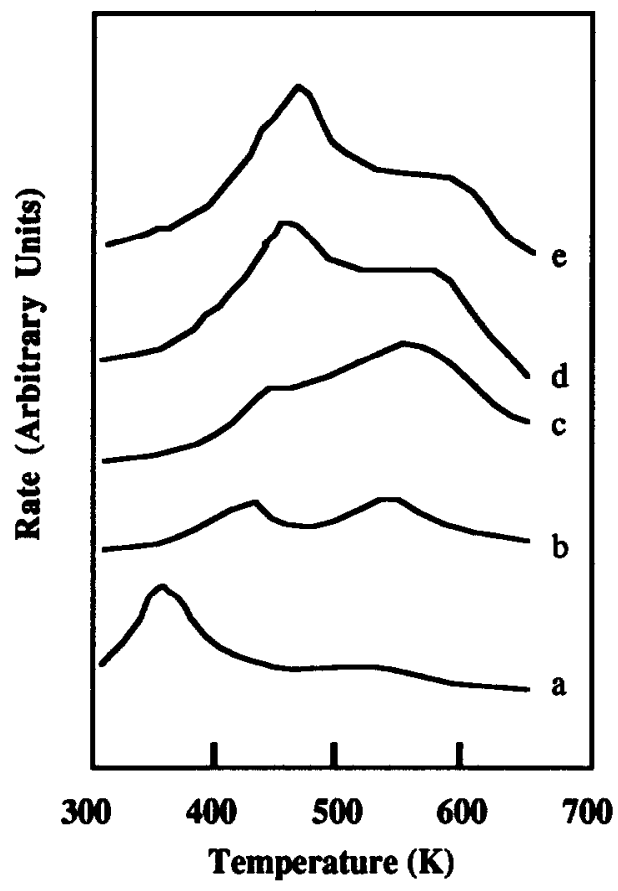

Figure 3. TPD spectra for MoN-4 with heating and flow rates of:
(a) $15 \mathrm{~K} / \mathrm{min}$ and $40 \mathrm{ccm}$,
(b) $16 \mathrm{~K} / \mathrm{min}$ and $20 \mathrm{ccm}$,
(c) $15 \mathrm{~K} / \mathrm{min}$ and $10 \mathrm{ccm}$,
(d) $25 \mathrm{~K} / \mathrm{min}$ and $10 \mathrm{ccm}$ and
(e) $35 \mathrm{~K} / \mathrm{min}$ and $10 \mathrm{ccm}$.

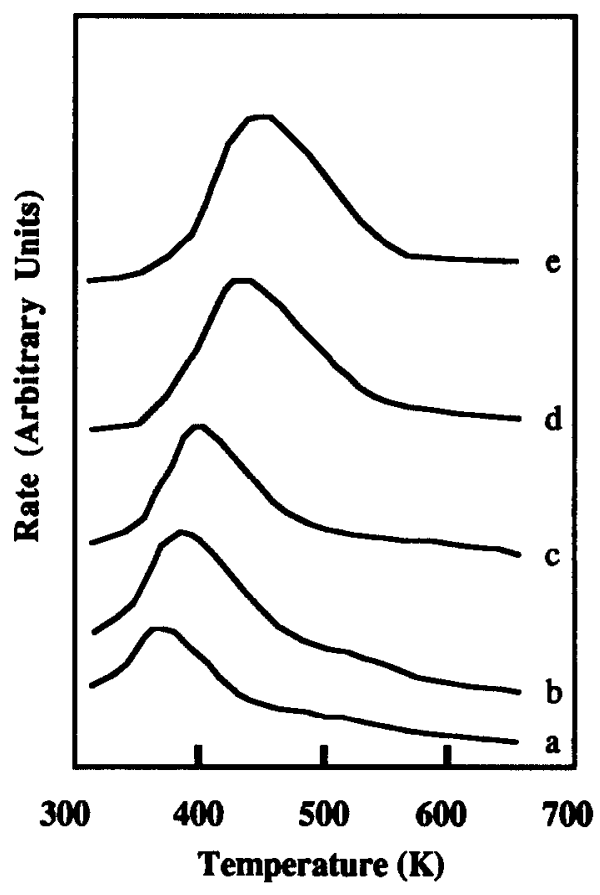

Figure 4. TPD spectra for MoN-8 with heating and flow rates of:

(a) $15 \mathrm{~K} / \mathrm{min}$ and $40 \mathrm{ccm}$,

(b) $15 \mathrm{~K} / \mathrm{min}$ and $20 \mathrm{ccm}$,

(c) $15 \mathrm{~K} / \mathrm{min}$ and $10 \mathrm{ccm}$,

(d) $25 \mathrm{~K} / \mathrm{min}$ and $10 \mathrm{ccm}$ and

(e) $35 \mathrm{~K} / \mathrm{min}$ and $10 \mathrm{ccm}$.

The symmetry of the desorption peak provided some information concerning the order of the rate-limiting process. For MoN-8 the desorption peak was consistently asymmetric suggesting that the rate limiting step leading to desorption was first order [23]. In the other catalysts where the peaks appeared to be more symmetric, the rate-limiting step may have been second order. Perhaps $\mathrm{H}_{2}$ desorption occurred in two steps:

$$
\mathrm{H}+\underset{*}{\mathrm{H}} \longrightarrow \mathrm{H}_{2}+* \longrightarrow \mathrm{H}_{2}+2 *
$$

The first reaction, combination of adsorbed atomic hydrogen, may have been rate-limiting in most of the catalysts. For MoN-8, the diffusion of atomic hydrogen may have been rapid enough or the surface occupied with enough atomic hydrogen so that the first order desorption of $\mathrm{H}_{2}$ was rate limiting. The latter reasoning is consistent with the proposition of Haddix et al. [20] that hydrogen adsorbed in rafts. 
Table 5

Summary of TPD Results

\begin{tabular}{|c|c|c|c|}
\hline $\begin{array}{l}\text { Catalyst } \\
\text { Code }\end{array}$ & $\underset{\pi_{L}}{\text { Temperature }}$ & Maximum & $\underset{\pi / S}{\text { Rate }(K)^{*}}$ \\
\hline $\begin{array}{l}\text { MoN-1 } \\
\text { MoN-2 } \\
\text { MoN-3 } \\
\text { MoN-4 } \\
\text { MoN-5 } \\
\text { MoN-6 } \\
\text { MoN-7 } \\
\text { MoN-8 }\end{array}$ & $\begin{array}{l}390 \mathrm{~s}^{\dagger} \\
356 \mathrm{vw} \\
348 \mathrm{~m} \\
452 \mathrm{vw} \\
391 \mathrm{~m} \\
373 \mathrm{vw} \\
357 \mathrm{vw} \\
397 \mathrm{vs}\end{array}$ & $\begin{array}{l}510 \mathrm{~m} \\
584 \mathrm{~s} \\
565 \mathrm{~m} \\
\\
565 \mathrm{~s} \\
551 \mathrm{~s}\end{array}$ & $\begin{array}{l}570 \mathrm{~m} \\
566 \mathrm{w} \\
492 \mathrm{~m} \\
591 \mathrm{~g}\end{array}$ \\
\hline
\end{tabular}

* From TPD spectra collected using a heating rate of $15 \mathrm{~K} / \mathrm{min}$ and Ar flow rate of $10 \mathrm{cc} / \mathrm{min}$.

$\dagger$ Designations indicate the relative intensity of the desorption peak: $v s=v e r y$ strong, $s=$ strong, $\mathrm{m}=$ medium, $\mathrm{w}=$ weak and $\mathrm{vw}=\mathrm{very}$ weak.

\subsection{High Reeolution Transmission Electron Microscopy}

Transmission electron microscopy was used to image the near surface structure of the Mo nitride catalysts. It should be noted that the catalysts were examined after passivation. Nevertheless, the near surface structure of the passivated catalyst was expected to be similar to that of the virgin, unpassivated catalyst given the results of Markel and Van Zee [17]. Figure 5 shows a HRTEM image taken of MoN-2 and its associated optical transform (diffraction pattern). The structure was clearly resolved looking down a $<100\rangle$ type zone axis (interplanar spacing $\sim 0.26 \mathrm{~nm}$ ). Crystallite sizes determined using HRTEM were similar to those estimated using XRD line broadening analysis. Furthermore, the particle sizes were consistent with average particle sizes determined from the BET surface area measurements.

The lattice constants were determined from fast Fourier transforms of selected sections near the edge of the crystalline domains. Lattice constants listed in Table 6 were calculated based on either an assumed face-centered or a body-centered unit cell [24]. Body-centered structures were more likely since they were self consistent with respect to both the $<001>$ and $<111>$ zone axes. Fitting to an hcp structure would require a lattice constant on the order of $3 \mathrm{~A}$ thus hcp structures were eliminated from consideration.

In all cases, the derived lattice constants and structures differed from that of bulk $\gamma-\mathrm{Mog}_{2} \mathrm{~N}$, which was the predominant phase identified by $\mathrm{x}$-ray diffraction analyses. Since the structure image was taken from regions within $\leq 50 \mathrm{~A}$ of the catalyst surface, the results suggested that the near surface structure differed significantly from that of the bulk. The known body-centered Mo nitride structures are $\mathrm{Mo}$ (bcc) and $\beta-\mathrm{Mo}_{16} \mathrm{~N}_{7}$ (bct). The $\beta-\mathrm{Mo}_{16} \mathrm{~N}_{7}$ phase is an interstitial compound capable of dissolving varying amounts of nitrogen. Nitrogen also has a finite solubility in Mo metal. The standard lattice constants for $\beta-M_{0}{ }_{16} \mathrm{~N}_{7}\left(a_{0}=4.19 \mathrm{~A}\right)$ and $\mathrm{Mo}_{0}\left(\mathrm{a}_{0}=3.15 \mathrm{~A}\right)$ bracketed those determined for the Mo nitrides. While the existence of metastable structures 
can not be ruled out, the results were consistent with the presence of substoichiometric $\beta-M_{16} \mathrm{~N}_{7}$ or mixtures of $\mathrm{Mo}$ and $\beta-\mathrm{Mo}_{16} \mathrm{~N}_{7}$ near the catalyst surfaces.

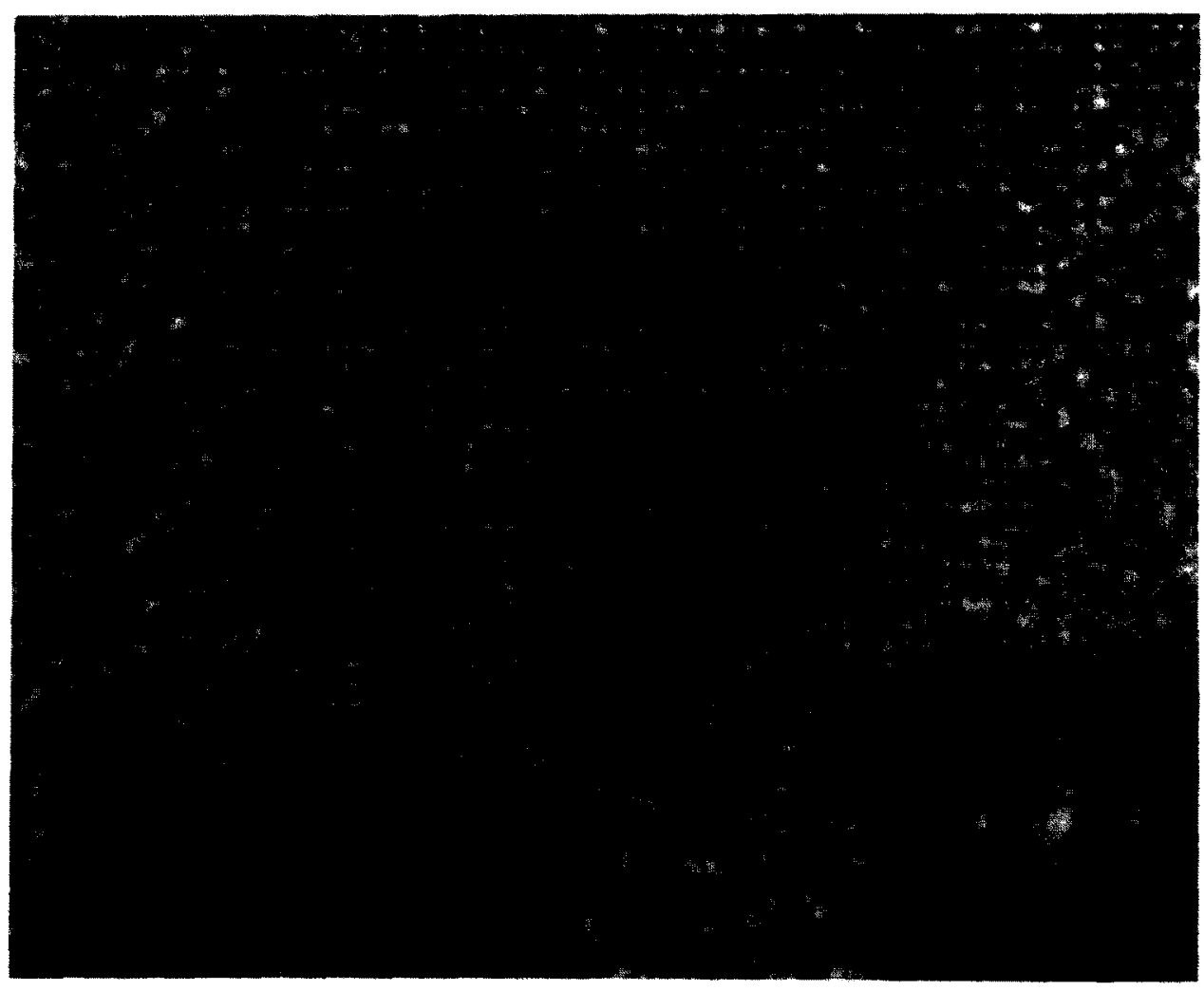

Figure 5. Atomic resolution transmission electron micrograph of MoN-2 looking down a $<100>$ zone axis. The inset shows the corresponding optical diffraction pattern.

The lattice constant decreased with increasing crystallite size. For Mo nitrides, like other interstitial compounds, the lattice constant can be considered a measure of the amount of interstitial nitrogen or oxygen. The smaller, near surface crystallites may contain higher concentrations of interstitial atoms than the larger near surface crystallites. This proposition is consistent with the XPS results as will be discussed in the next section. It should also be noted that there were distinct groupings with respect to the distortion from the basic cubic structure $(c / a=1-1.02$ and $c / a=1.05-1.15)$. Assuming a body-centered lattice, the latter group would be consistent with the presence of $\beta-M_{016} \mathrm{~N}_{7}$. The extent of the distortions increased with increasing lattice constant and decreasing particle size. These results may be indicative of significant differences between the N:Mo stoichiometries. 
Table 6

Lattice Constants Determined by Fourier Transform

Analysis of the High Resolution Transmission

Electron Micrographs

\begin{tabular}{cccc}
$\begin{array}{c}\text { Catalyst } \\
\text { Code }\end{array}$ & $\begin{array}{c}\mathrm{a}_{\mathrm{fc}} \\
(\AA)\end{array}$ & $\begin{array}{c}\mathrm{a}_{\mathrm{f}} \\
(\AA)\end{array}$ & $\mathrm{c} / \mathrm{a}$ \\
\hline MoN-1 & 4.76 & 3.28 & 1.05 \\
MoN-2 & 5.49 & 3.85 & 1.08 \\
MoN-3 & 5.28 & 3.73 & 1.15 \\
MoN-4 & 4.32 & 3.05 & 1.02 \\
MoN-5 & 4.85 & 3.43 & 1.02 \\
MoN-6 & 4.47 & 3.16 & 1.02 \\
MoN-7 & 4.61 & 3.26 & 1.02 \\
MoN-8 & 4.96 & 3.51 & 1.14 \\
\hline
\end{tabular}

\subsection{X-ray Photoelectron Spectroscopy}

The Mo(3d) spectra typically consisted of two envelopes which were deconvoluted to estimate the distribution of Mo oxidation states (Figure 6). The curve fitting was constrained so that the peaks for each $\mathrm{Mo}\left(3 \mathrm{~d}_{5 / 2}\right)-\mathrm{Mo}\left(3 \mathrm{~d}_{3 / 2}\right)$ doublet had the same shape and width (FWHM). The relative intensities of spin-orbit doublet peaks are given by the ratio of their degeneracies [25]. For the $\mathrm{Mo}\left(3 \mathrm{~d}_{5 / 2}\right)-\mathrm{Mo}\left(3 \mathrm{~d}_{3 / 2}\right)$ doublet the intensity ratio should be $\mathrm{I}\left(3 \mathrm{~d}_{5 / 2}\right) / \mathrm{I}\left(3 \mathrm{~d}_{3 / 2}\right)=3 / 2$ with a doublet splitting of $-3.15 \mathrm{eV}$. An important consequence of the deconvolution was a species with a $\mathrm{Mo}\left(3 \mathrm{~d}_{5 / 2}\right)$ binding energy of $228.6 \mathrm{eV}$. The binding energy of this species was midway between those assigned to $\mathrm{Mo}^{4+}$ $(230.0 \pm 0.2 \mathrm{eV})$ and $\mathrm{Mo}^{0}(227.8 \pm 0.2 \mathrm{eV})$. While it has been suggested that binding energies near $228.9 \mathrm{eV}$ are characteristic of $\mathrm{Mo}^{2+}$, there are no reliable standards [26]. However, the binding energy of this species was similar to that of $\mathrm{Mo}^{2+}$ in $\mathrm{Mo}_{2} \mathrm{C}$ [27]. We denoted this species as $\mathrm{Mo}^{\delta+}$ where $0<\delta<4$.

Table 7 summarizes the XPS results for the catalysts examined. The atomic compositions were estimated based on comparisons of the integrated peak intensities normalized by the atomic sensitivity factors. The N/Mo ratios were estimated using the $\mathrm{Mo}\left(3 \mathrm{p}_{3 / 2}\right)$ and $\mathrm{N}(1 \mathrm{~s})$ peak areas, while the $\mathrm{O} / \mathrm{Mo}$ ratios were estimated using the $\mathrm{Mo}\left(3 \mathrm{~d}_{5 / 2}\right)$ and $\mathrm{O}(1 \mathrm{~s})$ peak areas.

For most of the catalysts pretreatment in $\mathrm{H}_{2}$ at $673 \mathrm{~K}$ caused a significant reduction in the amount of oxygen and nitrogen near the surface. For MoN-8 pretreatment in $\mathrm{H}_{2}$ caused a decrease in the $\mathrm{O} / \mathrm{Mo}$ ratio but the $\mathrm{N} / \mathrm{Mo}$ ratio remained constant within experimental error. Furthermore the N/Mo ratio for MoN-8 was significantly higher than that of the other catalysts. Nitrogen to Mo stoichiometries of $\sim 0.5$ are consistent with the presence of either $\gamma-\mathrm{M}_{2} \mathrm{~N}$ or $\beta-\mathrm{Mo}_{16} \mathrm{~N}_{7}$ while $\mathrm{N} / \mathrm{Mo}$ ratios near unity indicate the presence of $\delta$-MoN. The presence of $\mathrm{MoN}$ at the surface of bulk $\gamma-\mathrm{Mo}_{2} \mathrm{~N}$ catalysts has been reported by Oyama et al. [28]. In an independent effort we synthesized a series of welldefined Mo nitride thin films. These films were prepared by either implanting $\mathrm{N}^{+}$ions into a Mo thin film $[29,30]$ or growing a Mo nitride film using ion beam assisted deposition. The N/Mo ratio for the $\delta$-MoN film was 1.16 and the Mo oxidation state distribution was similar to that of MoN-8. 


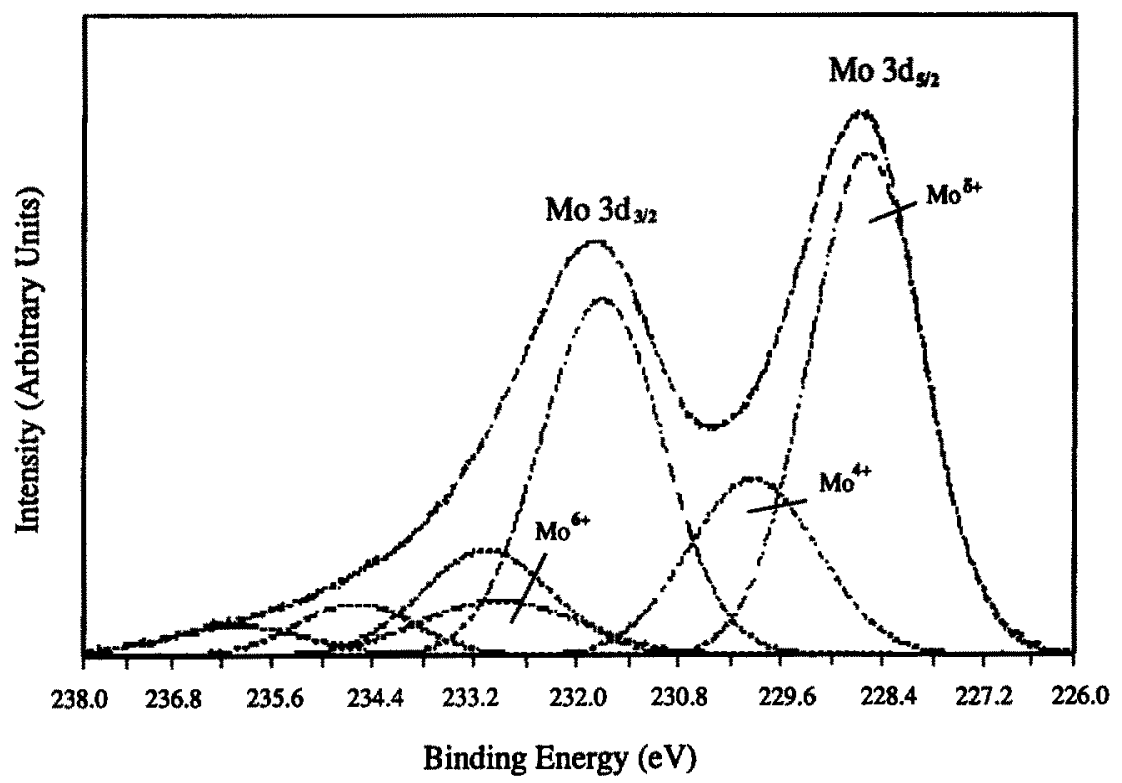

Figure 6. X-ray photoelectron spectrum of MoN-1 following pretreatment in $\mathrm{H}_{2}$ at $673 \mathrm{~K}$ for three hours. Spectral deconvolution was constrained so that the peaks for each $\mathrm{Mo}\left(3 \mathrm{~d}_{5 / 2}\right)-\mathrm{Mo}\left(3 \mathrm{~d}_{3 / 2}\right)$ doublet had the same FWHM, an intensity ratio of $3: 2$, and a splitting of $3.15 \pm 0.2 \mathrm{eV}$.

Table 7

Summary of the XPS Results

Catalyst Treatment O/Mo N/Mo

Code

Mo( $\left(3 d_{5 / 2}\right)$ Binding Energy (eV)

$\mathrm{Mo}^{0} \mathrm{Mo}^{\delta+} \mathrm{Mo}^{4+} \mathrm{Mo}^{6+}$

$\begin{array}{ccccccccc}\text { MoN-1 } & \text { passivated } & 0.69 & 1.04 & \ldots & 228.4(34) & 229.9(27) & 232.5(39) \\ & \text { reduced } & 0.18 & 0.48 & - & 228.4(53) & 230.1(37) & 232.5(10) \\ \text { MoN-2 } & \text { passivated } & 0.84 & 1.14 & - & 228.7(17) & 229.9(28) & 232.3(55) \\ & \text { reduced } & 0.20 & 0.60 & - & 228.5(43) & 230.1(44) & 232.6(13) \\ \text { MoN-4 } & \text { passivated } & 0.79 & 1.32 & - & 228.7(32) & 229.9(20) & 232.6(48) \\ & \text { reduced } & 0.11 & 0.63 & - & 228.7(69) & 229.9(20) & 232.4(11) \\ \text { MoN-8 } & \text { passivated } & 1.07 & 0.92 & 227.8(3) & 228.7(32) & 229.9(19) & 232.6(46) \\ & \text { reduced } & 0.13 & 0.91 & 227.8(4) & 228.8(57) & 229.9(29) & 232.5(10)\end{array}$

* Passivated at room temperature in $1 \% \mathrm{O}_{2}$ in He for 2 hours.

$\dagger$ Reduced in situ in $\mathrm{H}_{2}$ at $673 \mathrm{~K}$ for 3 hours. 


\section{DISCUSSION}

\subsection{Synthesie-Structure Relationships}

A prerequisite for the rational design and synthesis of Mo nitride catalysts is a detailed understanding of the relationship between their structural properties and the synthesis conditions. To this end we attempted to define the solid state reactions involved in the temperature programmed nitridation of $\mathrm{MoO}_{3}$. Solid state reactions typically involve processes including nucleation, growth and sintering. The rates for these processes are dependent on the temperature, reactant compositions and pressure. Temperature programmed reaction techniques can be used to balance the rates of nucleation, growth and sintering and alter the reaction pathways. Volpe and Boudart [31] and Jaggers et al. [11] proposed that the TPR of $\mathrm{MoO}_{3}$ with $\mathrm{NH}_{3}$ proceeded through two parallel reaction pathways; $\mathrm{MoO}_{3} \rightarrow \mathrm{MoO}_{\mathrm{x}} \mathrm{N}_{1-\mathrm{x}} \rightarrow \gamma-\mathrm{Mo}_{2} \mathrm{~N}$ and $\mathrm{MoO}_{3} \rightarrow>$ $\mathrm{MoO}_{2}->\mathrm{MoN}_{\mathrm{x}}$. The first reaction involves an fcc oxynitride intermediate. This reaction is considered topotactic since the lattices of the $\mathrm{MoO}_{\mathrm{x}} \mathrm{N}_{1 . \times}$ and $\psi$ Mo2 N phases have well defined crystallographic orientations relative to $\mathrm{MoO}_{3}$. It has been suggested that a key consequence of this topotactic reaction is the production of high surface area Mo nitride powders [11, 21]. The second proposed reaction involving $\mathrm{MoO}_{2}$ is not topotactic and is believed to produce lower surface area nitrides. Our results are consistent with existence of parallel reaction pathways, however, we believe that other intermediates were also involved in the conversion of $\mathrm{MoO}_{3}$ to $\mathrm{Mo}$ nitrides.

Two solid state reaction sequences were devised to account for the variations in product structure caused by varying the synthesis conditions. The first sequence which accounts for the TPR of $\mathrm{MOO}_{3}$ using an $\mathrm{NH}_{3}$ molar hourly space velocity of $17 \mathrm{hr}^{-1}$ (Figure 7a) is similar to that proposed by Jaggers et al. [11]. Differences between the properties of catalysts produced using different first heating rates suggested the presence of at least two intermediates. These intermediates may have been structurally and/or compositionally different. It has been proposed that topotactic reactions are required for the production of high surface area nitrides thus we inferred that the intermediate generated using the low first heating rate was a hydrogen bronze. The bronze, $\mathrm{H}_{\mathbf{x}} \mathrm{MoO}_{3}$, can be formed by the insertion of hydrogen between the loosely held double-thick layers of $\mathrm{MoO}_{6}$ octahedra in $\mathrm{MoO}_{3}$. This transformation can occur at temperatures near $650 \mathrm{~K}$. Subsequent reactions leading to the high surface area nitrides could involve the formation of Mo oxynitrides. Molybdenum dioxide is proposed as an intermediate during the synthesis of both medium and low surface area nitrides. Recall that $\mathrm{MoO}_{2}$ was detected in MoN-3, a medium surface area nitride.

Preliminary results from experiments using $\mathrm{H}_{\mathrm{x}} \mathrm{MoO}_{3}$ and $\mathrm{MoO}_{2}$ as primary reactants were consistent with the proposed reaction scheme. During these experiments the solid reactants were quickly heated to $723 \mathrm{~K}$ in flowing He then from 723 to $973 \mathrm{~K}$ at a rate of 100 or $200 \mathrm{~K} / \mathrm{hr}$ in flowing $\mathrm{NH}_{3}$. This permitted an independent examination of the influence of the second heating rate on the transformations of $\mathrm{H}_{\mathrm{x}} \mathrm{MoO}_{3}$ and $\mathrm{MoO}_{2}$. The bronze, $\mathrm{H}_{\mathrm{x}} \mathrm{MoO}_{3}$, produced a much higher surface area nitride than did $\mathrm{MoO}_{2}$. Molybdenum nitrides with surface areas up to $100 \mathrm{~m}^{2} / \mathrm{gr}$ were produced using $\mathrm{H}_{\mathrm{x}} \mathrm{MoO}_{3}$. Furthermore, increasing the heating rate resulted in a lower surface area for the TPR of $\mathrm{MoO}_{2}$. However contrary to our proposition the slower heating rate resulted in the highest surface area for the TPR of $\mathrm{H}_{\mathbf{x}} \mathrm{MoO}_{3}$.

As described earlier the space velocity had a significant influence on the structural properties of the Mo nitrides. We reasoned that the lower space velocity permitted longer exposures of the catalysts to $\mathrm{H}_{2} \mathrm{O}$. Water vapor can 
cause hydrothermal sintering or inhibit the topotactic transformations required to produce the high surface area catalysts. Products suffering only from sintering should have similar compositions. This was not the case. In situations where the space velocity was the only variable, the products had different structural and compositional characters. Thus we concluded that a different set of solid state reactions were involved in the TPR of $\mathrm{MoO}_{3}$ at low space velocities.

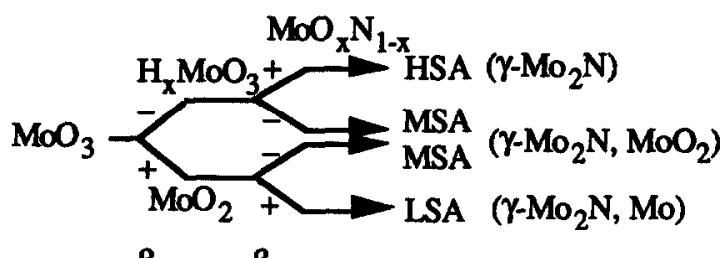

$\beta_{1} \quad \beta_{2}$

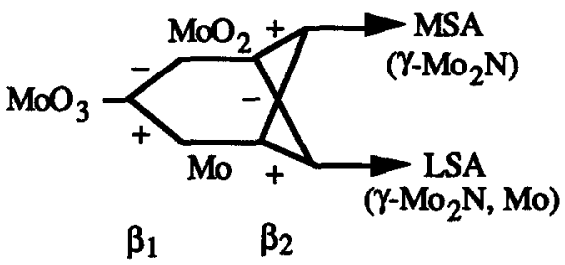

b

Figure 7. Solid state reaction schemes for the TPR of $\mathrm{MoO}_{3}$ with $\mathrm{NH}_{3}$ using the (a) high space velocity and (b) low space velocity (LSA, MSA and HSA denote low, medium and high surface area, respectively).

Figure $7 \mathrm{~b}$ illustrates a reaction scheme that accounts for structural properties of Mo nitrides synthesized using a space velocity of $8.5 \mathrm{hr}^{-1}$. We believe that the intermediate generated using the low first heating rate was $\mathrm{MoO}_{2}$ because of the similar and unique structural properties of the medium surface area nitrides. In particular the $\gamma-\mathrm{Mo}_{2} \mathrm{~N}$ crystallite sizes for all of the medium surface area nitrides were greater than $100 \AA$ while the crystallites sizes for the high and low surface area nitrides were $\sim 80 \AA$. The presence of Mo metal in both of the low surface area catalysts was taken as evidence of another intermediate. Potential origins of the Mo include production during a reaction parallel to reactions leading to the nitrides, or production as an intermediate during the formation of the nitride. If the Mo was produced during a separate reaction one might expect the presence of Mo in some of the other nitrides. Because MoN-5 and MoN-8 were the only nitrides where crystalline Mo was observed, we concluded that Mo was an intermediate. An intriguing ramification of this proposition is that the Mo:O stoichiometry and/or the Mo oxidation state of the intermediate governed the structural properties: $\mathrm{H}_{\mathrm{x}} \mathrm{MoO}_{3}$ leading to high surface area nitrides, $\mathrm{MoO}_{2}$ leading to medium surface area nitrides and Mo leading to low surface area nitrides. Our current efforts use in situ x-ray diffraction to elucidate additional features concerning the solid state reactions involved in the TPR of $\mathrm{MoO}_{3}$. In addition we are studying the influence of the precursor character on the properties of the resulting Mo nitrides. 
Differences between the crystallite sizes can be explained based on differences between the rates of nucleation and growth. Nucleation rates are favored by lower temperatures while growth rates are accelerated by using higher temperatures. In addition, nucleation is predominant for short reaction times due to the very low surface areas of the precipitates. For temperature programmed reactions the time that the materials are held at a given temperature should also be considered. Consequently we compared the product crystallite sizes based on the convolution of temperature and time. The higher heating rates should cause a decrease in the crystallite size for solid state transformations controlled by nucleation processes. Conversely, the crystallite size is expected to increase with increasing heating rate for transformations controlled by growth processes. On these bases we concluded that the nitriding of $\mathrm{H}_{\mathrm{x}} \mathrm{MoO}_{3}$ and $\mathrm{Mo}$ were controlled by the rate of nucleation while the nitriding of $\mathrm{MoO}_{2}$ was controlled by the growth rate.

\subsection{Structure-Function Relationships}

The concept of structure-sensitivity for Mo nitrides must not only include variations in the activities of differing crystallographic planes and phases but also variations in activities of surfaces with differing $\mathrm{N}: \mathrm{Mo}$ stoichiometries. The results of investigations using TPD, HRTEM and XPS clearly indicated that the characters of the Mo nitride surfaces were different. In this section we will identify trends that might assist in the development of detailed relationships between the structure, stoichiometry and function of Mo nitride HDN catalysts (see Table 8).

Features that distinguished MoN-8, the catalyst with the highest activity, from most of the other catalysts included a low surface area and concomitantly very large particles, the presence of small quantities of Mo metal, and the production of a single peak in the TPD spectrum. Since both MoN-8 and MoN5 , catalysts with widely differing catalytic properties, contained Mo metal we do not believe that the presence of Mo metal was responsible for the high activity of MoN-8. The XPS results also indicated the presence of only very small amounts of Mo metal at the surface of MoN-8. Nevertheless, this conclusion is tentative given that Mo is known to be active for HDS [32], a reaction that shares characteristics with HDN.

The relationship between activity and particle size, despite some scatter, was nearly linear with the activity increasing with increasing particle size. We also noticed that differences between the particle and crystallite sizes tracked with the activities. Deviations between the particle and crystallite sizes implicated the presence of polycrystalline particles and grain boundaries. Catalysts that consisted of polycrystalline particles possessed the highest activities. This result suggested that characteristics associated with the grain boundaries influenced the catalytic activities. On further inspection the activity was almost proportional to $\mathrm{d}_{p} / \mathrm{d}_{\mathrm{c}}^{2}$, a quantity that is expected to be proportional to the surface grain boundary length. We have consequently concluded that active sites resided along grain boundaries. These did not appear to be the only types of sites. Catalysts in which the particle and crystallite sizes were similar had similar albeit low activities. Thus we propose that at least two types of sites existed in the Mo nitrides; high activity sites along grain boundaries and low activity sites on particle surfaces. The TPD results which indicated the presence of two types of surface sites were qualitatively consistent with this proposition. Recall that for MoN-8, which had the highest activity and was presumed to have the greatest grain boundary length, only a single low temperature TPD peak was produced. Low 
temperature $\mathrm{H}_{2}$ desorption sites might reside along grain boundaries or other surface irregularities, and high temperature sites on the particle surface.

Table 8

Summary of Catalytic, Structural and Compositional Properties

\begin{tabular}{ccccccccc}
\hline $\begin{array}{c}\text { Catalyst } \\
\text { Code }\end{array}$ & $\begin{array}{c}\text { Normalized } \\
\text { Activity }\end{array}$ & $\begin{array}{c}\text { Particle } \\
\text { Size (A) }\end{array}$ & $\begin{array}{c}\text { Crystallite } \\
\text { Size }(\AA)\end{array}$ & $\begin{array}{c}\text { Low Tmax } \\
\text { TPD Peak }\end{array}$ & $\begin{array}{c}\text { abc } \\
(\AA)\end{array}$ & c/a & $\begin{array}{c}\text { N/Mot } \\
\text { Ratio }\end{array}$ \\
\hline MoN-8 & 100 & 1596 & 80 & vs & 3.51 & 1.14 & 0.91 \\
MoN-1 & 50 & 266 & 97 & s & 3.28 & 1.05 & 0.48 \\
MoN-7 & 43 & 140 & 132 & vw & 3.26 & 1.02 & \\
MoN-6 & 28 & 226 & 142 & vw & 3.16 & 1.02 & \\
MoN-3 & 19 & 145 & 108 & m & 3.73 & 1.15 & \\
MoN-5 & 19 & 532 & 78 & m & 3.43 & 1.02 & \\
MoN-4 & 17 & 123 & 130 & vw & 3.05 & 1.02 & 0.60 \\
MoN-2 & 15 & 55 & 77 & vw & 3.85 & 1.08 & 0.63 \\
\hline
\end{tabular}

* Based on reaction rates normalized by $\mathrm{O}_{2}$ uptake.

$\dagger$ Following reduction in flowing $\mathrm{H}_{2}$ at $673 \mathrm{~K}$.

Relationships between the activity and the surface structure and composition appeared more complex. The activity increased to a maximum then decreased with increasing lattice constant beyond $\sim 3.5 \AA$. To a first approximation the lattice constant should be a measure of the amount of interstitial nitrogen present in the lattice. The highest activity catalyst, MoN8, had the highest N:Mo stoichiometry. In fact the N:Mo ratio of unity for MoN-8 was distinctly different from that of the other catalysts. Given the other information about MoN-8, we concluded that nitrogen was concentrated at the grain boundaries perhaps in the form of MoN. Grain boundaries are characterized by a high degree of irregularity and reactivity. It is plausible that grain boundaries near or at the gas-solid interface provided nucleation sites for the conversion of $\gamma-\mathrm{Mo}_{2} \mathrm{~N}$ to $\mathrm{MoN}$. The presence of $\mathrm{MoN}$ could account for the high activity and somewhat unique adsorptive properties of MoN-8.

\section{CONCLUSIONS}

The structural properties of the Mo nitrides were complex functions of the synthesis conditions. In general the use of the low space velocities resulted in the production of low surface area materials while high space velocities led to high surface area materials. This behavior has been tentatively attributed to inhibition by $\mathrm{H}_{2} \mathrm{O}$ of the reaction pathways that are required for the production of high surface area Mo nitrides. Two solid state reaction sequences were devised to account for the structural characteristics of the high, medium and low surface area nitrides. The reaction sequence proposed to account for the production of high surface area nitrides involves topotactic transformations as has been suggested by Jaggers et al. [11] and Volpe et al. [31]. 
The Mo nitrides were as much as an order of magnitude more active for pyridine $\mathrm{HDN}$ than a commercial sulfided $\mathrm{Co}-\mathrm{Mo} / \mathrm{Al}_{2} \mathrm{O}_{3}$ catalyst. Furthermore, the selectivity for C-N bond hydrogenolysis over C-C bond hydrogenolysis was higher for the nitride catalysts. The product distributions for the Mo nitrides were similar, however, their specific activities were significantly different. Features that appeared to correlate with the activity were the particle size and grain boundary length, suggesting that HDN was structure-sensitive over the Mo nitrides. The high activity catalysts had low surface areas, large particles and significant grain boundary lengths. We proposed that two types of catalytic sites were present on the Mo nitrides. The highest activity sites appeared to be associated with the grain boundaries while lower activity sites resided on the particles. The $\mathrm{H}_{2}$ adsorption/desorption patterns qualitatively paralleled the activity patterns. Two types of surface desorption sites were present in most of the catalysts, and some also had subsurface sites. The low energy surface sites have been preliminarily attributed to the grain boundaries. Our results also suggested that $-21 \%$ of the surface Mo atoms were capable of chemisorbing oxygen (assuming a Mo:O ratio of unity) and the equivalent of $-5 \%$ of a monolayer of oxygen diffused into the nitride subsurface at $298 \mathrm{~K}$.

Stoichiometries determined using XPS suggested that MoN was present at the surface of the highest activity catalyst. Furthermore, our results suggested that MoN may have concentrated at the grain boundaries. It is well known that grain boundaries provide sites for preferential segregation. In all cases the surface structures were markedly different from the bulk structure. The predominant bulk phase in the Mo nitrides was $\gamma$-Mo2N. Small amounts of $\mathrm{MoO}_{2}$ and $\mathrm{Mo}$ were also detected in the medium and low surface area nitrides, respectively. The surface structures were consistent with the presence of either non-stoichiometric $\beta-\mathrm{MO}_{16} \mathrm{~N}_{7}$ or mixtures of $\mathrm{Mo}$ and $\beta$ $\mathrm{Mo}_{16} \mathrm{~N}_{7}$.

In conclusion, important details concerning the active species in Mo nitride HDN catalysts have been elucidated. This type of information will be useful in the rational design of Mo nitride catalysts for commercial applications.

\section{REFHRENCES}

1. J.S. Lee, L. Volpe, F.H. Ribeiro and M. Boudart, J. Catal. 112 (1988) 44.

2. S.T. Oyama, J.C. Schlatter, J.E. Metcalfe and J.M. Lambert, Jr., Ind. Eng. Chem. Res. 27 (1988) 1639.

3. J.S. Lee, S.T. Oyama and M. Boudart, J. Catal. 106 (1987) 125.

4. D.J. Sajkowski and S.T. Oyama, ACS Prep. Div. Petrol. Chem. 35(2) (1990) 233.

5. J.C. Schlatter, S.T. Oyama, J.E. Metcalfe and J.M. Lambert, Ind. Eng. Chem. Res. 27 (1988) 1648.

6. L. Leclercq, K. Imura, S. Yoshida, T. Barbee and M. Boudart, Preparation of Catalysts II, B. Delmon ed., Elsevier, NY, 627 (1978).

7. L. Volpe and M. Boudart, J. Phys. Chem. 90 (1986) 4874.

8. L. Leclercq, M. Provost, H. Pastor, J. Grimblot, A.M. Hardy, L. Gengembre and G. Leclercq, J. Catal. 117 (1989) 371.

9. R.B. Levy and M. Boudart, Science 181 (1973) 547.

10. J.H. Sinfelt and D.J.C. Yates, Nature Phys. Sci. 229 (1971) 27.

11. C.H. Jaggers, J.N. Michaels and A.M. Stacy, Chem. Mater. 2 (1990) 150.

12. L. Volpe, S.T. Oyama and M. Boudart, Preparation of Catalysts III, G. Poncelet, P. Grange and P.A. Jacobs eds., 147 (1983). 
13. B.E. Concha and C.H. Bartholomew, J. Catal. 79 (1983) 327.

14. S.J. Tauster, T.A. Pecoraro and R.R. Chianelli, J. Catal. 63 (1980) 515.

15. B.D. Cullity, Elements of X-ray Diffraction, Addison-Wesley Publishing Co., Massachusetts, 1978.

16. A.A. Reeves, Optimized Fast Hartley Transform for the MC68000 with Applications in Image Processing, MS Thesis, Thayer School of Engineering, Dartmouth College, Hanover, New Hampshire, 1990.

17. E.J. Markel and J.W. Van Zee, J. Catal. 126 (19y0) 643.

18. K.J. Leary, J.N. Michaels and A.M. Stacy, J. Catal. 107 (1987) 343.

19. G.W. Haddix, D.H. Jones, J.A. Reimer and A.T. Bell, J. Catal. 112 (1988) 556.

20. G.W. Haddix, J.A. Reimer and A.T. Bell, J. Catal. 108 (1987) 50.

21. M. Boudart and L. Volpe, J. Solid State Chem. 59 (1985) 332.

22. K.J. Leary, J.N. Michaels and A.M. Stacy, AIChE J. 34(2) (1988) 263.

23. J.L. Falconer and J.A. Schwartz, Catal. Rev.-Sci. Eng. $25(2)$ (1983) 141.

24. C.S. Barrett, Structure of Metals: Crystallographic Methods, Principles and Data, 3rd ed., Pergamon Press, 301 (1980).

25. D. Briggs and M.P. Seah, Practical Surface Analysis by Auger and X-ray Photoelectron Spectroscopy, John Wiley and Sons, New York, 1983.

26. J. Goldwasser, S.M. Fang, M. Houalla and W.K. Hall, J. Catal. 115 (1989) 34.

27. W.C. Moshier, G.D. Davis and G.O. Cote, J. Electrochem. Soc. 136 (1989) 356.

28. S.T. Oyama and M. Boudart, J. Res. Inst. Catal., Hokkaido Univ. 28(3) (1980) 305.

29. J-G Choi, D. Choi and L.T. Thompson, J. Mater. Res. 7(2) (1992) 374.

30. D. Choi, J-G Choi and L.T. Thompson, submitted for publication to Chem. Mater. (1992).

31. L. Volpe and M. Boudart, J. Solid State Chem. 59 (1985) 332.

32. A.J. Gellman, D. Neiman and G.A. Somorjai, J. Catal. 107 (1987) 92. 